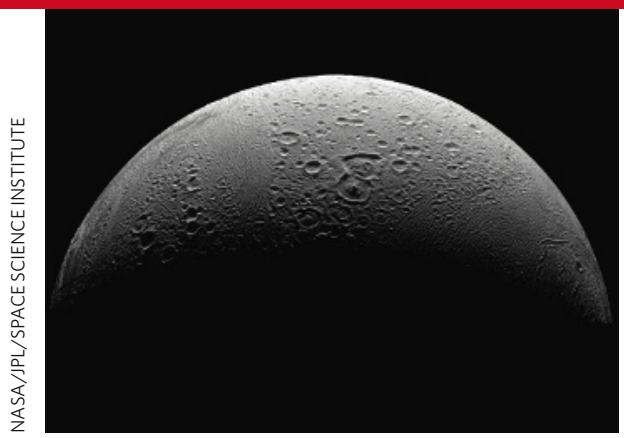

This image of Enceladus was captured last week.

\section{Saturn flyby is a success despite computer glitch}

NASA's Cassini spacecraft swooped past Enceladus, a tiny moon of Saturn, as planned on 12 March. But the dust analyser onboard failed just as the probe flew through the ice and gas plume that spews from the moon's south pole (see Nature 452, 139; 2008).

The glitch arose two hours before the flyby, when the team responsible for the instrument tried to upload a software patch to speed up its counting rate to as high as 100 particles per second.

Uploading the patch took only a few microseconds, but unfortunately coincided with a higher-priority command from the main spacecraft. The patch fizzled. "It's really frustrating," says Sascha Kempf, deputy principal investigator for the instrument at the Max Planck Institute for Nuclear Physics in Heidelberg, Germany. Kempf says the team can update the software in time for the next flyby in August.

The 11 other data recorders on the spacecraft worked fine. "We're disappointed in the one instrument," says Dennis Matson, project scientist for the mission at the Jet Propulsion Laboratory in Pasadena, California. "But it's more than outweighed by all the great stuff we did get."

\section{Bonn to play host to dementia research centre}

The German science ministry is providing $€ 40$ million (US\$ 62 million) to set up a research centre in Bonn to study neurodegenerative conditions such as Alzheimer's disease. A further $€ 20$ million will be distributed to six partner organizations in locations such as Munich and Tübingen.

Some 300 staff are expected to work at the German Centre for Neurodegenerative Diseases. A detailed research programme will be agreed in the next few months.

The new centre will come under the umbrella of the Helmholtz Association, which runs 15 national research institutes. It will be the first new Helmholtz centre to be founded since the country's reunification in 1990.

\section{Korean institute inquiry prompts two retractions}

A team led by a senior South Korean scientist is retracting two papers, in Science and Nature Chemical Biology, following a university investigation into the research.

Tae Kook Kim, of the Korea Advanced Institute of Science and Technology (KAIST) in Daejeon, had reported a new method for imaging living cells that used magnetized nanoparticles (J. Won et al. Science 309, 121-125; 2005, and J. Won et al. Nature Chem. Biol. 2, 369-374; 2006). KAIST's investigation is ongoing, but last month it reported preliminary findings that the "scientific truth" of the papers was in question. Investigators are also probing whether misconduct is involved.

Terry Sheppard, editor of Nature Chemical Biology, says that the KAIST investigation team told him that the authors wanted to retract the paper. The retraction, he notes, will occur "as soon as possible". Science also confirms that the team is working to retract its paper. 